\section{Saúde na Agenda de Desenvolvimento pós-2015 das Nações Unidas}

\author{
Health in the post-2015 United Nations \\ Development Agenda
}

\author{
La salud en la Agenda de Desarrollo post 2015 de \\ las Naciones Unidas
}

\author{
${ }^{1}$ Centro de Relações \\ Internacionais em Saúde, \\ Fundação Oswaldo Cruz, \\ Rio de Janeiro, Brasil. \\ 2 Escola Nacional de Saúde \\ Pública Sergio Arouca, \\ Fundação Oswaldo Cruz, \\ Rio de Janeiro, Brasil. \\ 3 Vice-presidência de \\ Ambiente, Atenção e \\ Promoção da Saúde, \\ Fundação Oswaldo Cruz, \\ Rio de Janeiro, Brasil. \\ ${ }_{4}^{4}$ Diretoria Regional de \\ Brasília, Fundação Oswaldo \\ Cruz, Rio de Janeiro, Brasil. \\ 5 Instituto Oswaldo Cruz, \\ Fundação Oswaldo Cruz, \\ Rio de Janeiro, Brasil. \\ Correspondência \\ P. M. Buss \\ Centro de Relações \\ Internacionais em Saúde, \\ Fundação Oswaldo Cruz. \\ Av. Brasil 4365, Rio de \\ Janeiro, RJ 21040-360, Brasil. \\ buss@fiocruz.br
}

\begin{abstract}
This paper evaluates health as a Sustainable Development Goal (SDG) in the context of the Post-2015 Development Agenda, between 2012 and 2014. Health was part of the debate since the Millennium Summit and the MDGs (2000), and it also appears in the documents discussing the Post-2015 Agenda, from the Rio+20 to the Open Working Group (OWG), whose report was submitted to the General Assembly of the United Nations (UNGA) 2014-2015, and in the Global Consultation on Health and the HighLevel Panel of Eminent Persons reports. The Authors concluded that the treatment of health in all these documents is uniform. They point out that the scope of the health-related SDG is very comprehensive, but its targets are conceptually fragmented and reduced. They advocate their change as to include not only the idea of social determinants of health, but also targets in the field of public health, which were not included in the proposal of the OWG. They also warn that the global and national governance systems need to be reformed and advocate more participation of the civil society, which can influence diplomacy, which, in turn, will be responsible for the agreement signed at the UNGA in 2015.
\end{abstract}

Health Priorities; Sustainable Development; World Health
Paulo Marchiori Buss 1

Danielly de Paiva Magalhães 1

Andréia Faraoni Freitas Setti 1

Edmundo Gallo 2

Francisco de Abreu Franco Netto 3

Jorge Mesquita Huet Machado 4

Daniel Forsin Buss 5

\section{Resumo}

O artigo examina a presença da saúde como Objetivo do Desenvolvimento Sustentável (ODS) na Agenda do Desenvolvimento pós-2015, no processo transcorrido entre 2012 e 2014. Presente desde a Cúpula do Milênio e os ODM (2000), a saúde também aparece nos documentos que tratam da Agenda pós-2015, da Rio+20 ao documento do Grupo de Trabalho Aberto das Nações Unidas (OWG), submetido à Assembleia Geral das Nações Unidas (AGNU) 2014-2015, passando pela Consulta Global sobre Saúde e o Painel de Alto Nível de Pessoas Eminentes. Os autores concluem pela uniformidade com que a saúde aparece nestes documentos. Identificam a amplitude do enunciado do ODS Saúde, mas alertam para o fracionamento e redução conceitual de suas metas. Propõem mudanças nas mesmas, acrescentando a questão dos determinantes sociais da saúde e metas em saúde pública, ausentes na proposta do OWG. Alertam para a necessidade de reformas na governança global e nacional, bem como para a participação da sociedade civil e da influência que pode exercer sobre a diplomacia, responsável pelo pacto que será firmado na AGNU de 2015.

Prioridades em Saúde; Desenvolvimento Sustentável; Saúde Mundial 


\section{Introdução}

A saúde teve importante presença na Agenda do Milênio (2000-2015), constituindo três dos oito Objetivos de Desenvolvimento do Milênio (ODM), pactuados na Cúpula do Milênio do ano 2000 1: redução da mortalidade infantil, redução da mortalidade materna e combate ao HIV/AIDS e às doenças negligenciadas, entre elas malária e tuberculose. Contudo, os demais ODM (redução da pobreza; ensino básico universal; igualdade entre os sexos e autonomia das mulheres; sustentabilidade ambiental; e estabelecimento de uma parceria mundial para o desenvolvimento) seguramente têm impacto sobre a saúde, por serem todos reconhecidos como potencialmente capazes de atuar sobre alguns dos seus principais determinantes socioambientais.

No processo de definição da Agenda do Desenvolvimento pós-2015 e dos Objetivos do Desenvolvimento Sustentável (ODS) (Figura 1), a saúde continua sendo objeto de atenção, con- forme se pode depreender do exame do documento final da Conferência das Nações Unidas sobre Desenvolvimento Sustentável (Rio+20), O Futuro que Queremos 2 e das iniciativas políticas globais decorrentes da orientação da Assembleia Geral das Nações Unidas (AGNU) de 2012, que a inclui como um dos objetos das 11 Consultas Temáticas Globais das Nações Unidas sobre ODS (setembro de 2012 a março de 2013) e também como tema das negociações intergovernamentais em andamento no âmbito da Organização das Nações Unidas (ONU), na qualidade de provável ODS.

Neste artigo será analisada a inserção da saúde na Agenda pós-2015, com especial foco nas negociações que transcorreram entre a Rio+20 (2012) e a AGNU 2014, assim como as projeções até a AGNU 2015, quando está previsto o encerramento do processo, com a definição da Agenda e dos ODS. O artigo também tratará de explicitar as posições divergentes entre os que defendem a cobertura universal em saúde (UHC) como ODS

Figura 1

Processo de formulação da Agenda e dos Objetivos do Desenvolvimento Sustentável (ODS), definido pela Rio+20 e aprovado na Assembleia Geral das Nações Unidas (AGNU) 2012.

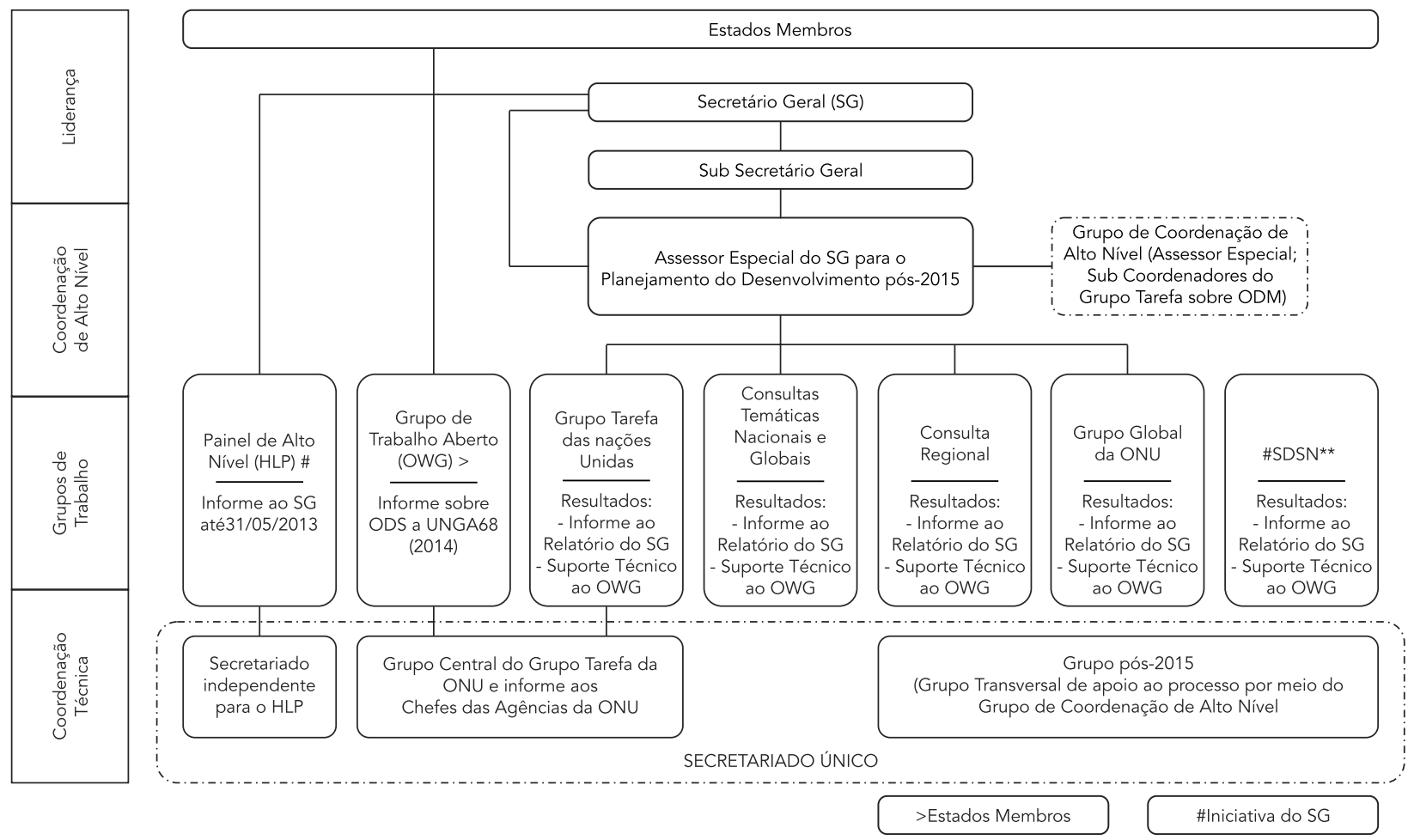


- basicamente a Organização Mundial da Saúde (OMS) e aliados - e aqueles que entendem que o ODS Saúde deveria ser mais amplo, como seria "assegurar vidas saudáveis e bem-estar para todos em todas as idades".

Finalmente, os autores discutem algumas das características propostas e as que consideram necessárias à governança global para o desenvolvimento e a consecução dos ODS, incluindo o de saúde, compreendendo que esta discussão transcende o interesse exclusivamente global, pelos impactos que os acordos internacionais firmados no âmbito das Nações Unidas têm sobre as políticas nacionais de desenvolvimento que, por sua vez, acabam por interferir significativamente na qualidade de vida das populações de todos os países do mundo.

\section{Metodologia}

Foi realizada análise dos documentos-chave da Agenda pós-2015 resultantes do complexo processo de formulação da Agenda e dos ODS, definido pela Rio+20 e aprovado na AGNU 2012, apresentado na Figura 1: a Declaração do Milênio do ano $2000^{1}$ e suas conexões com os demais ODM; o documento resultante da Rio+20, O Futuro que Queremos 2; o documento final da consulta global de Botswana sobre saúde na Agenda 3; o documento do Painel de Alto Nível (HighLevel Panel - HLP) 4; o documento da Rede de Soluções para o Desenvolvimento Sustentável (SDSN, em seu acrônimo em inglês) 5; e o documento resultante do Grupo de Trabalho intergovernamental da AGNU (UN Open Working Group on Sustainable Development - OWG) ${ }^{6}$. Também foram examinados os componentes de saúde nos informes das diversas consultas temáticas globais levadas a cabo pelas Nações Unidas 6, assim como realizada revisão bibliográfica de artigos e informes selecionados, produzidos por reconhecidos especialistas da área 7,8,9.

\section{Saúde e ODM}

Uma série de cúpulas e conferências realizadas pelas diversas agências das Nações Unidas durante a década de 1990 - que visavam "preparar o mundo para o século XXI" - culminaram, no ano 2000, com a Cúpula do Milênio e os Objetivos do Desenvolvimento do Milênio (ODM) 1. A entrada da saúde como ODM no ano 2000 advém da compreensão sobre a importância que têm populações saudáveis para o crescimento econômico e o desenvolvimento, por um lado e, por outro, do reconhecimento pela comuni- dade internacional das péssimas condições de saúde de grandes contingentes da população de países em desenvolvimento, o que se transformou em incontornável imperativo ético, a ser enfrentado também pelos países mais ricos. O mundo vivia, então, um período de estabilidade econômica, distante ainda da severa crise que se abateria sobre as economias centrais, a partir de 2007-2008, o que os levou a se comprometerem, à época, com a transferência de $0,7 \%$ dos respectivos PIB para projetos de desenvolvimento em países mais pobres. Contudo, a ajuda oficial para o desenvolvimento (ODA) alcançou em 2012 nos países ricos em média apenas $0,29 \%$ do PIB e, ademais, muito poucos países efetivamente cumpriram até aqui o compromisso assumido no ano 200010.

Segundo o Informe Final da Consulta Global sobre Saúde 3, “os ODM levaram a saúde ao mais alto nível político global, mobilizaram a sociedade civil, ampliaram a ajuda para o desenvolvimento em saúde, e contribuíram para melhorias consideráveis nos resultados de saúde em países de renda média e baixa". Contudo, o mesmo Informe chama a atenção de que "os ODM também contribuíram para abordagens fragmentadas do desenvolvimento: entre os diferentes ODM relativos à saúde, entre os ODM de saúde e outros ODM (...) e entre os ODM e outras prioridades omitidas da Agenda do Milênio".

Por outro lado, especialistas 7,9 também admitem que os ODM Saúde foram limitados, focando em questões muito específicas, que conduziram a programas verticais, com restrito efeito sobre o que seria de fato importante para a sustentabilidade de qualquer processo de transformação: a organização sustentável de sistemas nacionais de saúde, capazes de responder às necessidades das realidades sócio-sanitárias nacionais. Não se trata de desmerecer a importância dos três ODM Saúde, mas chamar a atenção de que o reforço de sistemas de saúde universais, integrais, equitativos e de qualidade (incluindo os ODM Saúde), seguramente traríam maior impacto, no curto (até 2015) e no longo prazo, sobre a saúde da população dos países em desenvolvimento.

Autores como Legge 9 alertam que os ODM ficaram longe de representar uma agenda do desenvolvimento, algo muito mais complexo do que elencar um conjunto de objetivos estreitos, ignorando que o verdadeiro desafio do desenvolvimento está na superação das múltiplas complexidades da globalização neoliberal, no que tem nossa inteira concordância.

Finalmente, o desempenho insatisfatório dos ODM Saúde, com metas não alcançadas globalmente e em diversos países 10, levou o Conselho Econômico e Social das Nações Unidas 
(ECOSOC), em uma de suas reuniões de alto nível de 2014 11, a propor dois caminhos complementares: "transformações estruturais necessárias para obter um crescimento centrado no emprego, a equidade distributiva, e marcos coerentes de política social que abordem as desigualdades nos planos nacionais e internacional" e, simultaneamente, a permanência dos ODM Saúde entre as metas do ODS Saúde, o que se constituiria em parcela da chamada "agenda inconclusa" dos compromissos do Milênio.

\section{Saúde no documento da Rio+20: O Futuro que Queremos 2}

Os Chefes de Estado reunidos na Rio+20 renovaram - ainda que, para muitos, apenas retoricamente - o "compromisso com o desenvolvimento sustentável e com a promoção de um futuro econômico, social e ambientalmente sustentável para o planeta e para as atuais e futuras gerações" 2. Tomando a erradicação da pobreza e da fome como o maior desafio global e requisito indispensável para o desenvolvimento sustentável, debruçam-se inevitavelmente também sobre o tema da saúde.

Assim, dos 286 parágrafos que compõem a declaração 2, dedicam nove deles (138 a 146) ao tema "saúde e população", no capítulo sobre "Áreas temáticas e temas intersetoriais”. O documento afirma, logo de início, que "a saúde é, simultaneamente, uma condição prévia, um resultado e um indicador de todas as três dimensões do desenvolvimento sustentável [social, econômica e ambiental]". Coerente com tal assertiva, identifica a necessidade de se implementar " $m e$ didas sobre os determinantes sociais e ambientais da saúde (...) a fim de criar sociedades inclusivas, equitativas, economicamente produtivas e saudáveis" e que "torne-se plenamente efetivo o direito de todos a desfrutar do mais alto nível possível de saúde física e mental".

Ao referir-se às medidas necessárias a este fim, os Chefes de Estado identificam a importância de reforçar sistemas de saúde para proporcionar "cobertura sanitária universal e equitativa", assim como convocam os "agentes pertinentes para participar de uma ação multisetorial coordenada, a fim de atender as necessidades de saúde da população mundial".

Integra a preocupação dos signatários do documento questões já contempladas pelos ODM: HIV, malária, tuberculose e as doenças tropicais negligenciadas, além da poliomielite, gripe e outras doenças transmissíveis e, pela primeira vez, a carga e a ameaça das enfermidades não transmissíveis, nomeadamente o câncer, as do- enças cardiovasculares, as respiratórias crônicas e a diabetes.

Na específica conexão entre saúde e poluição ambiental o documento destaca a importância de reduzir, entre outras coisas, a contaminação da atmosfera e da água e aquela causada por produtos químicos que afetam a saúde.

Tomar em consideração as questões demográficas e as migrações no planejamento do desenvolvimento nacional e a saúde sexual e reprodutiva, incluindo métodos seguros de planejamento familiar, também aparecem no segmento saúde e população do documento O Futuro que Queremos. E, finalmente, a aplicação dos dispositivos do Acordo TRIPS 12, que outorga flexibilidade para a proteção da saúde pública e, em particular, para promover o acesso universal aos medicamentos.

Portanto, o documento mostra a sensibilidade, pelo menos retórica, dos Chefes de Estado com as questões da saúde e da ação multisetorial sobre os determinantes sociais para alcança-la, ao lado do fortalecimento de sistemas de saúde para a prática da "cobertura universal e equitativa” relacionada com a saúde individual - mas sem menção à integralidade e qualidade, atributos fundamentais dos sistemas de saúde, nem às políticas e instrumentos da saúde pública/saúde coletiva, omissões graves do documento, que serão discutidas mais adiante.

Duas instâncias importantes se originaram da Rio+20. Um deles, o Fórum Político de Alto Nível (High-Level Political Forum) 13, definido como a principal plataforma das Nações Unidas para lidar com o desenvolvimento sustentável e que substitui a Comissão de Desenvolvimento Sustentável (CSD). O Fórum é constituído pelos Chefes de Estado de todos os países-membros das Nações Unidas, que se reúnem a cada quatro anos sob os auspícios da Assembleia Geral (primeira reunião em Setembro de 2013) 13 e, todos os anos, com representação de altos funcionários dos países, a exemplo dos Ministros das Relações Exteriores, sob os auspícios do ECOSOC (primeira reunião em Julho de 2014) 14. Foi instituído para "orientar a política, acompanhar a implementação, abordar desafios novos e emergentes $e$ incrementar a integração das dimensões económica, social e ambiental do desenvolvimento sustentável".

Outra instância foi o Comitê Inter-governamental de Especialistas sobre o Financiamento do Desenvolvimento Sustentável 15, com membros nomeados pelos grupos regionais das $\mathrm{Na}-$ ções Unidas, que teve a atribuição de identificar e estabelecer fontes estáveis de financiamento para o processo de desenvolvimento sustentável a ser implementado, particularmente em apoio 
aos países em desenvolvimento. O informe final 15 apresenta um quadro analítico para o financiamento do desenvolvimento sustentável, propõe uma série de políticas possíveis para subsidiar a escolha dos países, e sugere áreas em que a parceria global poderia contribuir para alcançá-lo.

Ainda como parte das discussões sobre a agenda pós-2015 e do seguimento da Rio+20, as cinco Comissões Regionais das Nações Unidas (inclusive a CEPAL, na América Latina e Caribe) organizaram consultas regionais recentes sobre o processo para alcançar o desenvolvimento sustentável, cujos resultados foram analisados por Chasek 16. No caso da América Latina e Caribe as consultas foram realizadas em Bogotá (Colômbia), em 201317.

\section{Consulta temática das Nações Unidas sobre saúde na Agenda do Desenvolvimento Pós-2015 3}

A Consulta temática das Nações Unidas sobre saúde na Agenda do Desenvolvimento Pós-2015 3 foi realizada por mandato da AGNU 2012, sob a coordenação da Suécia e Botswana, com a coparticipação da OMS, Fundo das Nações Unidas para a Infância (UNICEF) e Programa Conjunto das Nações Unidas sobre HIV e AIDS (UNAIDS), entre setembro de 2012 e março de 2013. Objetivava realizar ampla discussão nos níveis global, regional e nacional sobre progressos alcançados e lições aprendidas com os ODM Saúde; e estabelecer uma visão compartilhada entre Estados-Membros, agências das Nações Unidas, sociedade civil e outros atores-chaves sobre saúde na Agenda. A consulta foi presencial e virtual, com diversos documentos encaminhados por atores interessados 18. O documento final, Saúde na Agenda pós-2015 3 foi aprovado no Diálogo de Alto Nível sobre Saúde, culminância do processo, realizado em Gabarone (Botswana), em abril de 2013.

O referido documento trata das relações entre saúde e desenvolvimento, explicitando também uma visão sobre as relações entre saúde e os outros dez temas objetos das consultas globais realizadas no mesmo periodo: desigualdades; educação; crescimento e emprego; sustentabilidade ambiental; segurança alimentar e nutrição; governança; conflitos, violência e desastres; dinâmica populacional; água; e energia. Explora princípios, objetivos, metas, indicadores e abordagens para medição, implementação e monitoramento para um possível ODS Saúde.

O documento defende que o objetivo mais amplo da Agenda deve ser "bem-estar sustentá- vel para todos", para o qual a saúde daria uma "contribuição crítica" e do qual seria um "resultado fundamental”. Os princípios orientadores da nova Agenda devem incluir direitos humanos, equidade, igualdade de gêneros, prestação de contas (accountability) e sustentabilidade, priorizando os mais desfavorecidos, marginalizados, estigmatizados e as populações mais difíceis de alcançar. A equidade deve ser explicitada nas metas e indicadores, incluindo metas específicas para redução das desigualdades pré-existentes.

Segundo o documento, o ODS Saúde deve ser universal, já que os desafios em saúde também o são, ainda que as metas e indicadores devam ser adaptados às circunstâncias e as prioridades de saúde de cada país e a saúde deveria: (1) incluir metas específicas de saúde como parte de outros objetivos setoriais de desenvolvimento (saúde em todas as políticas); (2) estabelecer uma abordagem de saúde holística, cobrindo todo o curso da vida, com ênfase em promoção da saúde e prevenção de doenças; (3) acelerar o progresso onde as metas dos ODM ainda não foram alcançadas e estabelecer metas mais ambiciosas para o futuro; e (4) enfrentar a crescente carga das doenças não transmissíveis, doenças mentais e outros desafios emergentes em saúde.

Para cumprir esta pauta, o ODS específico de saúde seria "maximizar vidas saudáveis", no qual o setor saúde jogaria importante papel, embora longe de ser exclusivo, pois exigiria intervenções de todos os setores da sociedade.

Neste sentido (e guardando inteira coerência com a recomendação de uma "abordagem multisetorial” para a saúde, proposta no documento da Rio+20), o informe de Botswana sugere que maiores sinergias entre saúde e outros setores podem ser conseguidas por meio da formulação dos objetivos que, para serem alcançados, requerem coerência entre as políticas e soluções compartilhadas entre vários setores, ou seja, uma "abordagem que mobilize todo o governo" ou, em outras palavras, uma abordagem "saúde em todas as políticas”. São especificamente mencionados ODS em "determinantes da saúde" evidentes, como educação, igualdade de gêneros, energia sustentável, nutrição, água, saneamento e câmbio climático, que deveriam abrigar metas relacionadas com a saúde, o que veio a ocorrer no documento do OWG encaminhado à AGNU 2014, como se verá adiante.

O documento assume que a "agenda inconclusa”, representada pelos ODM não alcançados, assim como a saúde sexual e reprodutiva, universalização da vacinação e enfermidades não transmissíveis deveriam estar presentes como metas, sob o objetivo maior da "maximização de vidas saudáveis". 
Considera contribuição-chave do setor saúde a "cobertura e acesso universal" a um efetivo e acessível sistema de saúde integral (todo o continuum de cuidados) em todas as etapas da vida $\mathrm{e}$ que proteja a todos de riscos financeiros.

Os autores avaliam que, ainda que esta posição signifique um avanço em relação a sistemas universais, contempla apenas marginalmente a visão da produção social da saúde, predominando sua concepção biomédica. Por consequência, as propostas se concentram na assistência à saúde individual, com reduzida menção às dimensões da saúde pública, populacional e coletiva.

\section{Saúde no documento do Painel de Alto Nível 4}

O Painel de Alto Nível de Pessoas Eminentes, composto por 30 personalidades de diversas regiões do mundo, oriundas de governos, academia, sociedade civil e do setor privado, sob a coordenação dos Presidentes da Libéria e da Indonésia e do Primeiro Ministro do Reino Unido, foi instituído pelo Secretário Geral das Nações Unidas em setembro de 2012, com vistas a preparar um documento de contribuição aos debates sobre os ODS.

O Informe do Painel (maio de 2013) propõe uma agenda universal de erradicação da pobreza extrema até 2030, guiada por cinco "mudanças transformadoras" [transfomative shifts]: (a) Não deixar ninguém para trás (Leave no one) - Garantir que ninguém tenha seus direito humanos universais ou oportunidades econômicas básicas negadas e erradicar a pobreza extrema em todas as suas formas, além de assegurar um padrão básico de bem estar; (b) Colocar o desenvolvimento sustentável no centro - Integrar os três pilares do desenvolvimento sustentável, promovendo inclusão social e freando o ritmo da mudança climática e da degradação ambiental; (c) Transformar as economias para que gerem empregos e crescimento inclusivo - uma inflexão na direção de padrões sustentáveis de produção e consumo, que assegurem possibilidades de bons empregos e qualidade de vida, num mundo de recursos naturais limitados; (d) Construir a paz e instituições efetivas, transparentes e responsáveis para todos; (e) Forjar uma nova parceria global - promover parcerias capazes de promover solidariedade, cooperação e responsabilização conjunta.

O documento propõe "Assegurar vidas saudáveis" como ODS Saúde, no que coincide com o Informe de Botswana que lhe foi enviado como subsídio, além de outros 10 temas: acabar com a pobreza; Empoderar meninas e mulheres e al- cançar a igualdade de gênero; Prover educação de qualidade e de forma continuada; Assegurar a segurança alimentar e boa nutrição; Universalizar o acesso à água e ao saneamento; Assegurar energia sustentável; Gerar empregos, modos de vida sustentáveis e crescimento equitativo; Gerir os recursos naturais de forma sustentável; Garantir a boa governança e instituições eficazes; Garantir sociedades estáveis e pacíficas; e Criar um ambiente global favorável e catalisar recursos financeiros de longo prazo.

O ODS Saúde proposto constitui ampliação significativa em relação aos ODM e à cobertura universal de saúde, inicialmente proposta como o objetivo, depois como meta e, finalmente, aparecendo como meio, ainda que apresentada em uma visão restrita, como apontado mais a frente.

\section{Saúde na Rede de Soluções para o Desenvolvimento Sustentável (SDSN) 5}

A SDSN foi lançada pelo Secretário Geral das Nações Unidas em 2012 para "mobilizar conhecimento científico e tecnológico global sobre os desafios do desenvolvimento sustentável, incluindo o desenho e a implementação de uma agenda global pós-2015 sobre desenvolvimento sustentável". Após realizar consultas por meio do seu Conselho de Liderança e uma rede mais ampla de grupos temáticos sobre assuntos chave do desenvolvimento sustentável, a SDSN associa-se à visão da Rio+20 e o traduz como um "conceito holístico" que integra três dimensões da sociedade (econômica, social e ambiental), agregando às mesmas uma quarta dimensão, a "boa governança".

A SDNS definiu 12 Grupos Temáticos, incluindo a saúde, que reunem cientistas, engenheiros, acadêmicos, membros da sociedade civil e do setor privado. A versão preliminar do documento produzido pelo GT sobre Saúde propõe o enfrentamento da "agenda inconclusa" dos ODM, agregando uma abordagem de promoção da saúde, prevenção de enfermidades e cuidados à saúde nos diferentes estágios da vida, incluindo a prevenção controle das doenças transmissíveis (mas não se limitando à HIV/AIDS, TB e malária); DCNT, doenças mentais, lesões e deficiências; saúde da criança e do adolescente; direitos sexuais e reprodutivos, incluindo gravidez segura; atenção aos idosos; e serviços de emergências. Argumenta a favor da oferta e acesso sem barreiras a serviços de atenção primária de alta qualidade, inclusive de "serviços de saúde pública" - sem mencionar quais seriam; a vacinas, medicamentos e tecnologias essenciais de qualidade; e realça a importância do controle do tabagismo. 
Defende também a necessidade de recursos humanos capacitados para a cobertura universal, o que incluiria agentes comunitários de saúde. Para o financiamento de tal sistema propõe que o poder público dos países de renda baixa e média deve aplicar pelo menos $5 \%$ do PIB para a implementação da UHC e que, em apoio à mesma estratégia, os países ricos devem destinar pelo menos $0,1 \%$ do seu PIB (do total de $0,7 \%$ da ODA destinada à promoção do desenvolvimento sustentável).

Invoca a importância de adotar "políticas pró-saúde” em outros setores, particularmente nos sistemas de agricultura e alimentação; saneamento básico e redução da poluição do ar interior dos domicílios, do ar e da água; comércio e investimentos; políticas e transportes urbanos; e para a mitigação dos efeitos do câmbio climático sobre a saúde humana.

Paradoxalmente, apesar de inclinar-se no documento-raiz pela orientação de "alcançar bem-estar e saúde em todas as idades”, a maior parte do documento setorial é dedicada quase exclusivamente à questão da "cobertura universal em saúde".

\section{Saúde e Agenda pos-2015 no âmbito da OMS}

A saúde na Agenda pós-2015, como ocorreu com os ODM, tem ocupado espaços políticos e técnicos no Comitê Executivo da OMS e nas recentes Assembléias Mundiais da Saúde (AMS). Em maio de 2013, a AMS aprovou a resolução WHA66.11, na qual solicitava à Diretora Geral que promovesse ativamente o debate sobre a saúde na Agenda e apresentasse um informe. A OMS tem participado no processo em Nova York (Estados Unidos) por meio de sua oficina junto às Nações Unidas e da participação direta de funcionários em reuniões do OWG. Em Janeiro de 2014, o Secretariado apresentou documento no qual foi resumido o estado da arte e propostas ao Comitê Executivo, na sua 134a reunião (documento EB134/18). A 67a AMS, em maio de 2014 aprovou a resolução WHA67.14 na qual fica decidida a garantia de que a saúde tenha posição central na Agenda pós-2015.

O que tem notabilizado a posição da OMS no contexto da Agenda pós-2015 tem sido a defesa da cobertura universal em saúde, um objetivo eminentemente setorial, como ODS Saúde. Esta posição contrasta, na visão dos autores, com diversos posicionamentos históricos da OMS desde sua constituição como agência das Nações Unidas, como ocorreu na Conferência sobre Atenção Primária de Alma Ata (1978) e no amplo debate mundial sobre determinantes sociais da saúde (2008; 2011).

\section{Saúde no processo do OWG 6}

O OWG, previsto no documento final da Rio+20, é um grupo intergovernamental, instituído pela Resolução 67/555 da Assembleia Geral das Nações Unidas (AGNU) de 2012 para apresentar na AGNU de 2014 um documento que contenha propostas de ODS e suas metas principais. Trata-se do vértice do processo narrado nas páginas anteriores deste artigo; isto é, ao OWG chegaram os documentos das consultas-diálogos globais, do Painel de Alto Nível e de outros atores interessados, incluindo sociedade civil e setor privado.

O OWG tem “30 vagas”, cada uma delas liderada por dois países, constituindo-se num subconjunto dos 193 Estados-membros das Nações Unidas, que podem alternar-se na representação. É um espaço eminentemente diplomático, a ser influenciado por outras áreas dos respectivos governos e sociedade civil dos países. No caso do Brasil, por exemplo, foi instituído o Grupo de Trabalho Interministerial sobre a Agenda para o Desenvolvimento Pós-2015 20, composto pelos Ministérios das Relações Exteriores, do Meio Ambiente, do Desenvolvimento Social, da Fazenda e Secretaria Geral da Presidência da República, bem como por pontos focais de diversos Ministérios, inclusive o da Saúde, para contribuir na definição de posições do governo brasileiro no OWG.

Depois de realizadas as treze reuniões programadas entre março de 2013 e julho de 2014, após intensas negociações e posicionamentos de diversos países e grupos de países e "major groups" da sociedade civil 21,22, o OWG consensuou um Outcome Document 6, encaminhado ao exame da AGNU 2014, que o transformou no Documento A/68/970, considerando-o como a "principal base" para os debates subsequentes até a AGNU de 2015.

O documento chegou à UNGA 2014 com o aval do ECOSOC 14 e foi recebido com manifestações de apoio (e algumas restrições) de importantes segmentos de países, como são o G77+China 21, Grupo dos Países Árabes, União Africana, União Europeia e países como Estados Unidos, Brasil e outros, que tiveram em comum a recomendação de promover o menor número de emendas ao mesmo.

No documento, o OWG defende que a erradicação da pobreza e da fome, a promoção de padrões sustentáveis de produção e consumo e a proteção e gestão de recursos naturais são obje- 
tivos maiores e requerimentos essenciais para o desenvolvimento sustentável.

Reafirma compromissos com a Declaração Universal dos Direitos Humanos, liberdade, paz e segurança, direito ao desenvolvimento e a adequados padrões de vida, como direito à alimentação, respeito às leis, boa governança, equidade de gênero e empoderamento da mulher, assim como com sociedades democráticas.

Reconhece que existem diferentes abordagens, visões, modelos e ferramentas para que cada país possa alcançar o desenvolvimento sustentável em suas três dimensões, assim como a importância da cooperação internacional para o desenvolvimento.

O OWG propõe 17 ODS (Figura 2), cada um deles contendo metas (que totalizam 169) e indicadores. Dois deles (os ODS 16 e 17) são referentes à "meios de implementação" gerais dos ODS.

Entre os ODS está incluido o ODS Saúde, enunciado como "assegurar vidas saudáveis e promover o bem-estar para todos em todas as idades", que contém nove metas e quatro meios de implementação (Figura 3). Três das metas do ODS Saúde se referem à "agenda inconclusa" dos ODM Saúde: (1) redução da mortalidade materna; (2) acabar com a mortalidade previnível de recém-nascidos e crianças abaixo de cinco anos; e (3) acabar com as epidemias de HIV/AIDS, tuberculose, malaria e outras doenças "tropicais" negligenciadas, assim como combater as hepatites, as doenças transmitidas pela água e outras doenças transmissíveis.

As outras seis se referem a: (4) redução de mortes prematuras por doenças não-transmissíveis (DNT) e promoção da saúde mental e bemestar; (5) prevenção e tratamento do abuso de substâncias aditivas, incluindo estupefacientes, e o consumo nocivo de álcool; (6) redução das mortes e lesões por acidentes de trânsito; (7) garantia do acesso universal a serviços de saúde sexual e reprodutiva; (8) alcançar a cobertura universal de saúde, em particular a proteção contra riscos financeiros, o acesso a serviços de saúde essenciais de qualidade e o acesso a medicamentos e vacinas seguros, eficazes, acessíveis e de qualidade para todos; e (9) saúde ambiental, expressada na redução de mortes e enfermidades produzidas por produtos químicos perigosos e a contaminação do ar, água e solo.

Quatro meios de implementação específicos são propostos (Figura 3): (a) Aplicação do Convênio Marco da OMS para o Controle do Tabaco em todos os países; (b) Apoiar a pesquisa e desenvolvimento de vacinas e medicamentos para as doenças transmissíveis e não-transmissíveis que afetam

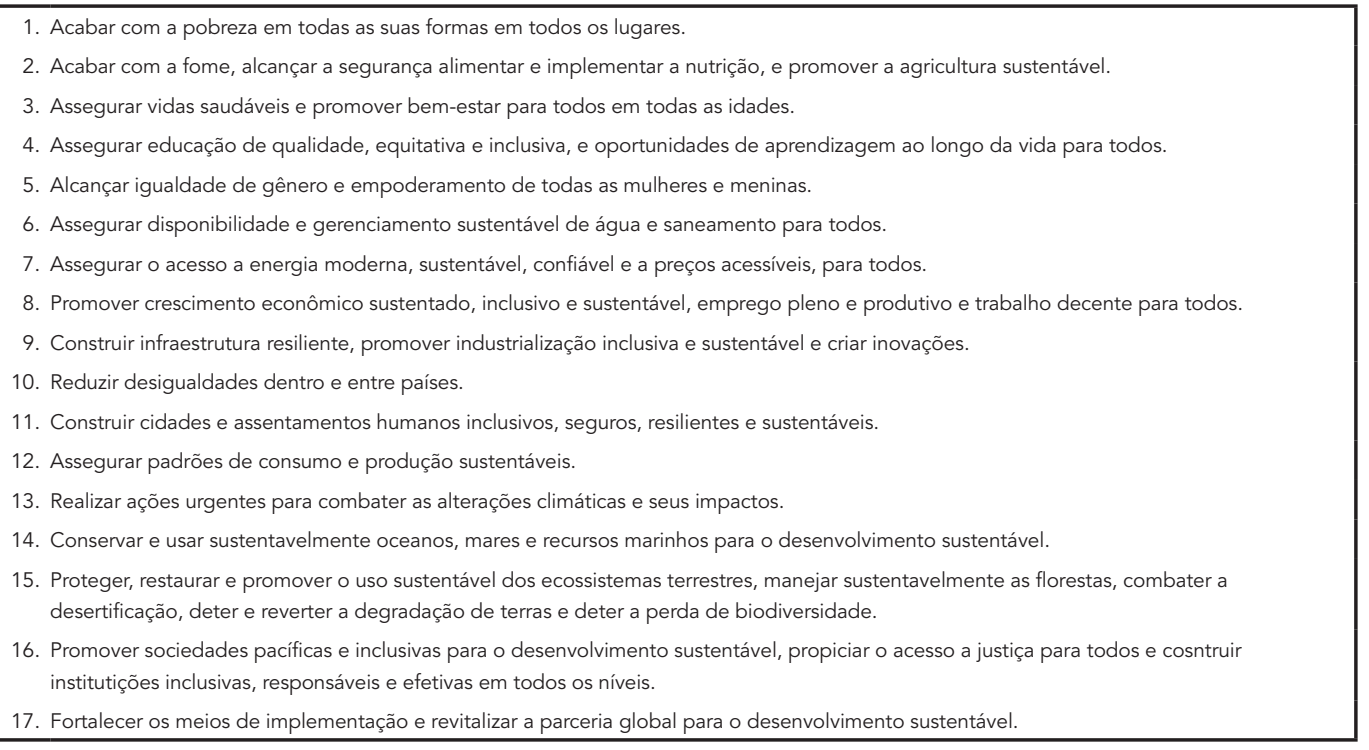

Fonte: http://sustainabledevelopment.un.org/focussdgs.html. 
Figura 3

Metas do ODS Saúde (Assegurar vidas saudáveis e promover bem-estar para todos em todas as idades). Grupo de Trabalho Aberto da Assembleia Geral das Nações Unidas (OWG).

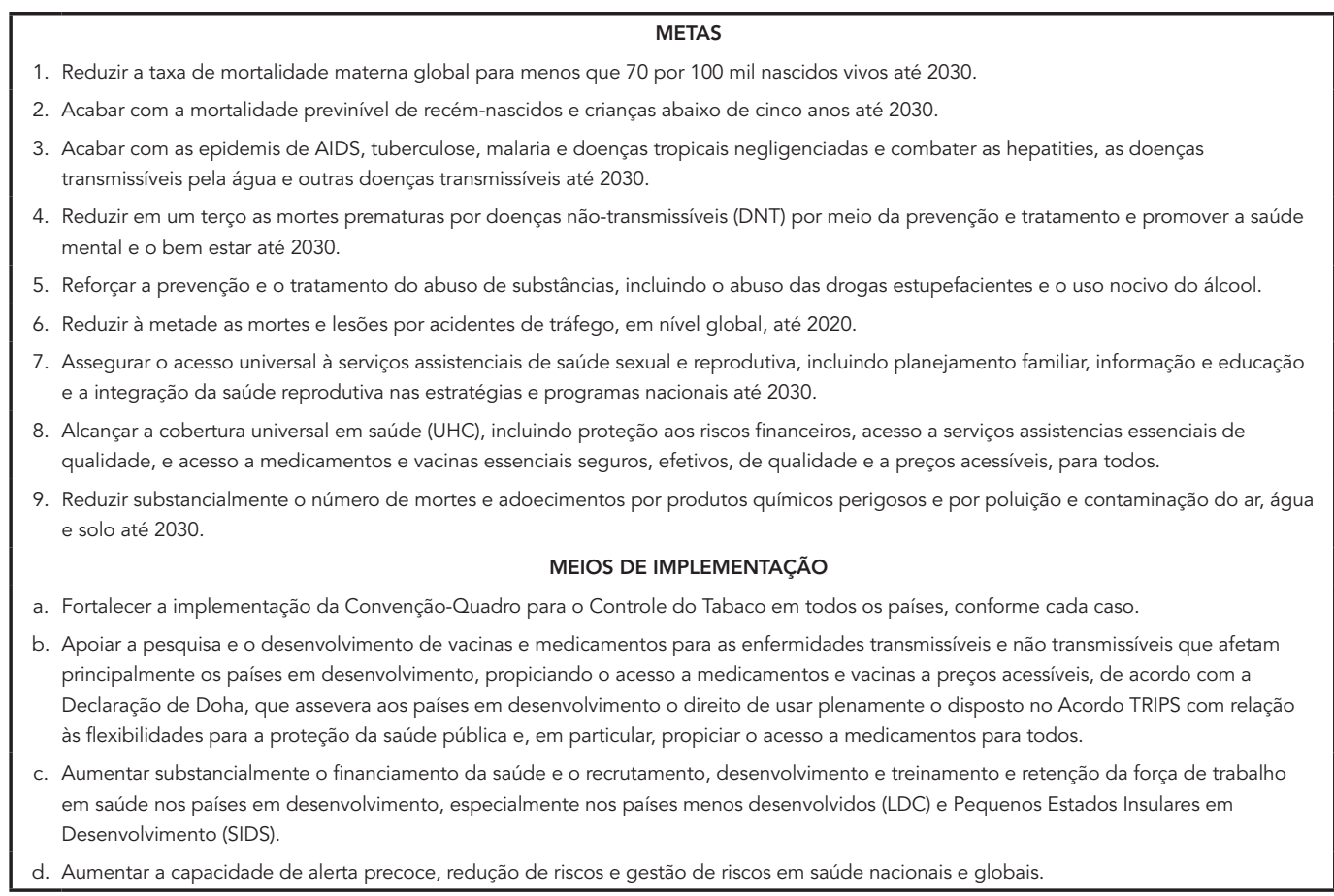

Fonte: http://sustainabledevelopment.un.org/focussdgs.html.

primordialmente aos países em desenvolvimento e facilitar o acesso a medicamentos e vacinas essenciais, de acordo com a Declaração de Doha sobre o Acordo TRIPS e a Saúde Pública; (c) Aumentar o financiamento da saúde e a contratação, desenvolvimento, capacitação e retenção de pessoal de saúde nos países em desenvolvimento; e (d) Reforçar a capacidade dos países de alerta precoce, redução de riscos e gestão dos mesmos na saúde nacional e mundial.

Dada à importância que outros ODS e respectivas metas têm para a saúde, bem como eventuais omissões nos mesmos, examinou-se também esta dimensão no documento do OWG. Assim, muitos dos outros 16 ODS (Figura 2) contêm metas diretamente relacionadas com saúde (por exemplo, eliminação da fome, segurança alimentar, nutrição segundo necesidades, redução da desnutrição, desenvolvimento infantil, acesso à agua de qualidade e saneamento com redução de doenças, desastres naturais e mortes, violência e saúde, poluições de ar, solo e água e suas influências sobre saúde). Neste sentido, o OWG incorporou, de alguma forma, as idéias da estratégia de "saúde em todas as políticas" quando define metas relacionadas com saúde em outros ODS que não o de saúde. Contudo, curiosamente metas que mencionem diretamente a saúde estão ausentes em ODS de "natureza econômica", como industrialização sustentável ou crescimento econômico e emprego decente. A proteção à saúde dos trabalhadores em ambientes potencialmente prejudiciais ou ações sobre poluição ambiental derivada dos processos produtivos, por exemplo, são ignorados nos ODS 8 e 9, expressando o divórcio entre questões econômicas e ambientais e saúde humana.

Um longo caminho ainda precisa ser trilhado para a superação destas omissões, buscando estabelecer maior compatibilidade e coerência entre os ODS, para alcançar um desenvolvimento sustentável que de fato considere seus três componentes: sociais, ambientais e econômicos.

Fica muito claro, ainda, que todos os ODS são, de alguma forma, "determinantes sociais da saúde", embora não mencionem diretamente a saúde entre suas metas. É o caso da eliminação da 
pobreza, da educação, da equidade de gênero, da energia sustentável, da infraestrutura, das inequidades, das cidades e assentamentos humanos, da produção e consumo sustentável e de ambientes sustentáveis e biodiversidade, por exemplo.

Embora, como se disse, cada ODS contém meios de implementação próprios e apropriados, os ODS 16 e 17 são interpretados como "meios de implementação gerais", pos se referem a questões de governança que cruzam transversalmente todos os demais, bem como contém outras questões gerais: tipos de instituições necessárias para o desenvolvimento sustentável, financiamento, tecnologias, "capacity builindg", comércio, parcerias para o desenvolvimento, temas sistêmicos (global $\mathrm{x}$ nacional x local; formulação e coordenação de políticas, entre outros) e dados para monitoramento e avaliação.

Com tudo isto, cresce de importância a elaboração de estratégias e meios e implementação "transversais", que articulem entre si todos os ODS propostos, em ações coerentes de governança, como políticas governamentais intersetoriais. A saúde humana - como produto social e um dos resultados esperados do desenvolvimento, como asseveram os Chefes de Estado e de Governo no documento da Rio+20 - é o exemplo mais gritante da importância de se mobilizar tais esquemas de governança, que incluem negociações inter-setoriais no interior dos governos e a participação de diferentes segmentos da sociedade civil.

\section{Discussão: saúde, ODS e governança}

O conteúdo do ODS Saúde, desde o documento final resultante da Rio+20 até o Documento Final do OWG 6 , que sintetiza os diversos processos percorridos ao longo dos últimos dois anos, teve desenvolvimento praticamente linear, isto é, pouco foi alterado na sua essência. Seu enunciado "Assegurar vidas saudáveis e bem-estar para todos em todas as idades" é um objetivo ambicioso, tanto do ponto de vista político-diplomático quanto sanitário, dentro do qual as metas reproduzem de forma um pouco mais detalhada - como seria de esperar - quase todo o conteúdo do segmento dedicado à saúde no documento resultante da Rio+20, exceto quanto à questão crítica dos determinantes sociais da saúde.

Embora na sua formulação geral o objetivo seja positivo, referindo-se à "saúde" relacionando-a com bem-estar, quase todas suas metas se referem à "redução de doenças e/ou mortes" e nunca à "promoção da saúde", o que seria uma inversão de paradigma, coerente com o enunciado mais amplo e positivo do ODS Saúde. No percurso do processo foi eliminada, por exemplo, a meta "au- mento da expectativa de vida saudável (EVS)", meta "positiva" proposta pela International Epidemiological Association (IEA) 23.

As três primeiras "metas objeto" do ODS Saúde pertencem à "agenda inconclusa" dos ODM: mortalidade materna (meta 1) - um dos grandes "fracassos" no processo de ODM Saúde por não ter sido alcançada na maioria dos países em desenvolvimento; "acabar" com as causas "previníveis" na morbidade e mortalidade neonatal e de crianças abaixo de 5 anos (meta 2); e reduzir a morbimortalidade por doenças como AIDS, tuberculose, malária e "outras" enfermidades "tropicais" negligenciadas (meta 3). A seguir, encontram-se quatro "metas objeto" relacionadas com as "novas ameaças", caso das DNT, incluindo doenças mentais (meta 4); uso de substâncias nocivas, lícitas e ilícitas (meta 5); doenças e mortes por acidentes de tráfego (meta 6); e as doenças de origem ambiental (meta 9).

As duas "metas instrumentais", acesso universal à serviços assistenciais de saúde sexual e reprodutiva (meta 7) e cobertura universal em saúde (meta 8), que se realizam no interior dos "sistemas de saúde", são profundamente dependentes da economia política global e nacional da saúde e não apenas de formulações técnicas de dirigentes da saúde. Isto implica na necessidade de comprometimento político profundo de outros setores das políticas e das regulações públicas, caso contrário estas metas se reduzirão a formulações retóricas que jamais se concretizarão. $\mathrm{Na}$ verdade, a realização de qualquer das metas propostas no ODS Saúde dependem de um elenco muito bem articulado de ações no interior de sistemas de saúde universais, equitativos, integrais e de qualidade, assim como sobre os múltiplos determinantes sociais da saúde que se localizam fora do setor saúde.

\section{Cobertura universal de saúde}

No seu marco de referência, o OWG designa a "cobertura universal em saúde" como "meta" (8), transformando-a do que seria um ODS Saúde apenas setorial e seguramente "minimalista" em uma meta necessária, mas não suficiente, para ambicionar o alcance de vidas saudáveis, pois aborda a questão do sistema de saúde apenas parcialmente (assistência aos enfermos, com ênfase no mecanismo dos seguros saúde). Por outro lado, o conceito de UHC utilizado é genérico, precisando de adjetivações que o expliquem, como discutiremos adiante.

Já o adjetivo “essenciais", presente na mesma meta, quando se refere ao acesso a medicamentos, vacinas e tecnologias, remete à idéia de "pacotes mínimos", determinados exclusivamente pela 
capacidade econômica dos países e não pelas necessidades de suas populações. A meta sobre saúde sexual e reprodutiva apoia-se aparentemente nas recomendações da Task Force da Conferência sobre População e Desenvolvimento (ICPD) 24 , documento especializado ao qual remetemos o leitor mais interessado nesta meta do ODS Saúde.

O debate sobre o ODS Saúde girava em torno de duas grandes tendências: uma, liderada pela OMS, privilegiando a UHC como ODS saúde e, outra, propondo um ODS saúde mais amplo e integrador, afinal resumido no enunciado "assegurar vidas saudáveis e bem-estar", que inclui UHC, além de exigir que se cumpram todos os demais ODS para alcança-lo. Ou seja, colocando "saúde" de fato no centro do desenvolvimento sustentável.

A OMS privilegiou em seus documentos preliminares 25 apenas a contribuição setorial da saúde aos ODS, enquanto os demais macroprocessos 2,3,4,5,6, trouxeram uma visão ampliada de saúde. A SDSN 5 colocou-se inicialmente numa posição ambígua, mas no documento setorial sobre saúde refere-se quase exclusivamente à UHC. Finalmente, o OWG ${ }^{6}$, alinhado com a tendência da maioria das instâncias, definiu "assegurar vida saudável e bem-estar para todos em todas as idades" como ODS Saúde.

Assume-se, neste artigo, a perspectiva da "saúde ampliada", pelas razões expostas a seguir. Em primeiro lugar, o ODS "assegurar vidas saudáveis e bem-estar" é muito mais amplo do que uma proposição de "cobertura universal em saúde", objetivo de responsabilidade exclusiva do setor saúde, sem demandar o comprometimento de outros setores. Certamente a UHC também contribui para o objetivo maior, na medida em que se propõe a tratar das pessoas enfermas, o que seguramente faz parte de uma melhor qualidade de vida e ajuda a prolongar a vida. Entretanto, UHC é necessária, mas não suficiente para "assegurar vidas saudáveis", pois são necessárias outras políticas e medidas que atuem sobre outros determinantes sócio-ambientais da saúde, que são focos de outros ODS, como educação de qualidade, acesso universal à água e saneamento, seguridade alimentar e nutricional, sociedades pacíficas, entre outros. Ademais, para "assegurar vidas saudáveis e bem-estar” é necessário abordar, com equidade, também outras dimensões dos determinantes socio-ambientais da saúde, sequer consideradas nos ODS mencionados para a futura Agenda. É possível, inclusive, que mesmo onde exista uma cobertura universal, a saúde - por ser uma "produção social" e não apenas resultante da biologia humana - se encontre estagnada ou declinante, justamente por causa da desconsi- deração à determinantes que se encontram fora do sistema de saúde.

Em segundo lugar, uma dimensão de saúde mais ampla como ODS, por ser de caráter nitidamente intersetorial, torna mais fácil compartilhar compromissos com outros ODS em prol da saúde na Agenda pós-2015, por meio de novos modelos de governança, que incluem objetivos compartilhados, planejamento conjunto e ações intersetoriais.

Um terceiro aspecto relevante do debate diz respeito aos componentes do conceito UHC 8 . Universal não significa necessariamente "equidade", "qualidade" ou "integralidade", atributos fundamentais da "saúde como direito", mas tão somente asseguramento para algum tipo de atenção, em geral "pacotes mínimos de serviços clínicos em atenção primária”, como dão a entender documentos que circulam veiculando o conceito. Cobertura não implica em acesso e utilização de serviços ou resposta às necessidades em saúde mas, em geral, apenas estar registrado em "alguma organização" que lhe garanta "algum tipo de atenção". Por outro lado, saúde pode simplesmente estar se referindo a atenção médica aos enfermos, sem compromissos com a integralidade, isto é o continuum de cuidados, incluindo o enfrentamento dos determinantes sociais da saúde. Por fim, sem equidade, corre-se o risco que os próprios sistemas de saúde sejam geradores de inequidades, pelas desigualdades no acesso e utilização. Portanto, ainda que se considerasse "saúde" como a "resposta setorial" adequada, certamente seria preciso ir além de "UHC" e, então, propor "sistemas de saúde baseados no direito à saúde e que sejam universais, equitativos, integrais e de qualidade".

Em quarto lugar, incorrendo nas mesmas omissões de outros planos globais para a saúde, como o Relatório do Banco Mundial de 1993, Investing in Health 26, as metas do ODS Saúde concentram-se na atenção individual, sem qualquer alusão às políticas do campo da saúde pública/ saúde coletiva, como: a vigilância sanitária, ferramenta fundamental para a regulação em saúde, na linha que delimita interesses do capital e da população; a vigilância epidemiológica, espaço especializado da saúde pública sobre a ocorrência dos problemas coletivos que demandam ação do sistema de saúde; a saúde ambiental, no contexto da saúde humana, animal e eco-sistemas; o planejamento e gestão em saúde, fundamental para a integralidade e outros atributos dos sistemas de saúde; a promoção da saúde; a educação em saúde; os determinantes políticos globais da saúde; ou a medição e ação sobre a os determinantes sociais e as inequidades em saúde; para citar apenas alguns dos campos omitidos. 
Proposição de alterações das metas do ODS Saúde (Assegurar vidas saudáveis e promover bem-estar para todos em todas as idades).

1. Reduzir a taxa de mortalidade materna global para menos que 70 por 100 mil nascidos vivos até 2030

2. Acabar com a mortalidade previnível de recém-nascidos e crianças abaixo de cinco anos até 2030.

3. Acabar com as epidemis de AIDS, tuberculose, malaria e doenças tropicais negligenciadas e combater as hepatities, as doenças transmissíveis pela água e outras doenças transmissíveis até 2030.

4. Reduzir em um terço as mortes prematuras por doenças não-transmissíveis (DNT) por meio da prevenção e tratamento e promover a saúde mental e o bem estar até 2030 .

5. Reforçar a prevenção e o tratamento do abuso de substâncias, incluindo o abuso das drogas estupefacientes e o uso nocivo do álcool.

6. Reduzir à metade as mortes e lesões por acidentes de tráfego, em nível global, até 2020.

7. Assegurar o acesso universal à serviços assistenciais de saúde sexual e reprodutiva, incluindo planejamento familiar, informação e educação e a integração da saúde reprodutiva nas estratégias e programas nacionais até 2030.

8. Alcançar a cobertura universal em saúde (UHC), incluindo proteção aos riscos financeiros, por meio de sistemas universais, equitativos, integrais e de qualidade, acesso a serviços assistencias e de saúde pública de qualidade necessários, e acesso a medicamentos e vacinas e tecnologias assistenciais e de saúde pública necessários seguros, efetivos, de qualidade e a preços acessíveis, para todos.

9. Reduzir substancialmente o número de mortes e adoecimentos por produtos químicos perigosos e por poluição e contaminação do ar, água e solo até 2030 , e proteger a saúde da população em relação a tais ameaças.

10. Enfrentar os determinantes sociais da saúde, por meio de formas inovadoras de governança que incluem os demais setores governamentais e a sociedade civil.

\section{MEIOS DE IMPLEMENTAÇÃO}

a. Fortalecer a implementação da Convenção-Quadro para o Controle do Tabaco em todos os países, conforme cada caso.

b. Apoiar a pesquisa e o desenvolvimento de vacinas e medicamentos e tecnologias assistenciais e de saúde pública necessárias para enfrentar as enfermidades transmissíveis e não transmissíveis que afetam principalmente os países em desenvolvimento, propiciando o acesso a medicamentos e vacinas a preços acessíveis, de acordo com a Declaração de Doha, que assevera aos países em desenvolvimento o direito de usar plenamente o disposto no Acordo TRIPS com relação às flexibilidades para a proteção da saúde pública e, em particular, propiciar o acesso a medicamentos para todos.

c. Aumentar substancialmente o financiamento da saúde e o recrutamento, desenvolvimento e treinamento e retenção da força de trabalho em saúde nos países em desenvolvimento, especialmente nos países menos desenvolvidos (LDC) e Pequenos Estados Insulares em Desenvolvimento (SIDS).

d. Aumentar a capacidade de alerta precoce, redução de riscos e gestão de riscos em saúde nacionais e globais, assim como a capacidade para vigilância sanitária e regulação.

Coerente com esta revisão crítica, faz-se o esforço de propor uma redação alternativa nas metas para o ODS Saúde (Figura 4) que, essencialmente, substitui UHC por sistemas de saúde universais, integrais, equitativos e de qualidade; e acrescenta o enfrentamento dos determinantes sociais da saúde por meio de formas inovadoras de governança que incluam os setores governamentais envolvidos e a sociedade civil. Propõe, ainda, ajustes em outras metas propostas.

\section{Governança}

As declarações e compromissos de Chefes de Estado e de outros atores globais com o desenvolvimento sustentável, ainda que extremamente importantes do ponto de vista político e também ideológico, podem quedar-se exclusivamente retóricos, se não forem acompanhados de transformações profundas na "governança do desen- volvimento", em âmbitos nacional e global. Em outra oportunidade já discutimos a governança em saúde e ambiente para o desenvolvimento sustentável, artigo 27 ao qual remetemos o leitor, no qual já apontavamos a importância da retomada do papel do Estado e do planejamento estratégico inter-setorial, com participação social, na condução e regulação do desenvolvimento.

Formulações sobre governança no âmbito do OWG, como já se mencionou, encontram-se nos ODS 16 (Alcançar instituições capazes e eficazes) e 17 (Fortalecer e melhorar os meios de implementação e a parceria global para o desenvolvimento sustentável). Tratam de princípios mais gerais (sete ao todo) e, para cada ODS, de meios específicos de implementação (46 ao todo). Entre as metas, elencam governança participativa; instituições capazes, efetivas e responsáveis; eliminação da corrupção; obediência às regras da lei, entre outros princípios gerais inerentes à 
governança. Mencionam ainda planos nacionais de desenvolvimento, para o que pedem o apoio à capacitação nos países em desenvolvimento, ainda que o planejamento intersetorial não chegue a ser mencionado, o que nos parece uma omissão a ser ainda superada.

No campo da ODA o documento insiste no cumprimento dos $0,7 \%$ do PIB por parte dos paises desenvolvidos, com a destinação de pelo menos $0,15 \%-0,20 \%$ para os menos desenvolvidos.

Insatisfeito com os meios de implementação propostos, o G77 21 - clube de cerca de 130 países em desenvolvimento que tem adotado posições internacionais comuns - apresentou um conjunto ampliado de meios em todos os ODS.

Os meios de implementação propostos para o ODS Saúde são apenas quatro, entre os 46 propostos no total, e referem-se a: tabaco; $\mathrm{P} \& \mathrm{D}$ em medicamentos e vacinas para enfermidades transmissíveis; cooperação com a indústria farmacêutica para o acesso a medicamentos essenciais a preços acessíveis; pessoal de saúde; e riscos nacionais e globais em saúde. Eles certamente não dão conta nem do amplo objetivo, nem das metas propostas. Meios de implementação diretamente relacionados com saúde, presentes nos demais ODS, sequer são mencionados no ODS Saúde e tampouco nos ODS onde estão localizados, omissão a ser considerada com urgência.

Como se vê, não estão propostos mecanismos consistentes de governança para o processo de desenvolvimento sustentável em geral, tampouco para a saúde, senão meios insuficientes de implementação de ODS isoladamente, o que contribui para sua fragmentação, prejudicando uma implementação coerente e harmônica, com co-responsabilidades, vitais num processo desta envergadura e complexidade.

Para superar estas omissões preocupantes, alguns exercícios deveriam ser desenvolvidos. Um excelente ensaio de planejamento intersetorial, seria monitorar e avaliar o impacto da execução (ou não) das metas de saúde presentes nos outros ODS e a repercussão das mesmas sobre a saúde. Em escala global, um grupo intersetorial de agências das Nações Unidas - que inclua a OMS - e, em escala nacional, um grupo que tenha o Ministério da Saúde em sua composição deveria implementar este processo. A OMS deveria dedicar parte significativa de sua atuação política e técnica em chamar a atenção para estes determinantes sociais, políticos e econômicos da saúde contidos nos ODS, incluindo o preparo de uma guia de planejamento intersetorial, oferecendo-o como contribuição à dimensão saúde no desenvolvimento sustentável.
O mesmo grupo deve considerar o já expressivo elenco de recomendações elaborado para uma nova governança da saúde nos últimos anos, seja em "saúde e desenvolvimento", "governança da saúde global" e "governança global e saúde", a serem consideradas: (1) o Informe Closing the Gap in $a$ Generation 28, resultante da Comissão Mundial sobre Determinantes Sociais da Saúde (DSS); (2) a Declaração Política do Rio sobre DSS 29, resultante da Conferência Mundial da OMS sobre DSS (Rio de Janeiro, 2011); (3) a Declaração de Helsinque sobre Saúde em Todas as Políticas ${ }^{30}$, resultante da Conferência Mundial sobre Promoção da Saúde (Helsinque, 2013); e (4) o Informe The Lancet-University of Oslo para o enfrentamento dos determinantes políticos globais na geração das inequidades em saúde 31 . O próprio documento dos Chefes de Estado na Rio+20 (O Futuro que Queremos, $\$$ 138) 2 recomenda "medidas sobre os determinantes sociais e ambientais da saúde", assim como propostas de novos modelos de formulação de políticas aparecem como recomendações do Forum Político de Alto Nível sobre desenvolvimento sustentável por meio do recém emitido $H L P F$ Issue Brief 5 com o tema From Silos to Integrated Policy Making 32.

\section{Conclusões}

Os resultados e propostas dos processos integrantes da definição da Agenda do Desenvolvimento pos-2015 e dos ODS desde a Rio+20 (Figura 1) tem a maioria de suas orientações semelhantes quanto a principios gerais, embora se verifiquem algumas diferenças, tanto nos ODS como um todo e respectivas metas, quanto no ODS Saúde.

Apesar da recomendação feita por inúmeros países e grupos de países, por ocasião do recebimento do documento na AGNU, em setembro de 2014, de que o mesmo recebesse o menor número de emendas e alterações possível até sua aprovação na AGNU 2015, é pouco provável que isto aconteça. Os inúmeros e conflitantes interesses de diferentes países, grupos de países, ONGs, empresas e setor privado e, mesmo, das próprias burocracias das Agências das Nações Unidas, quanto às orientações para o desenvolvimento sustentável até 2030 e seguintes, certamente imporão mudanças ao documento que afinal chegará para a assinatura dos Chefes de Estado e de Governo em 2015.

As radicais transformações da economia, do social e do ambiente que se aceleraram nos últimos anos apontam que no horizonte futuro de 15-20 anos as transformações se acelerarão ainda mais, com consequências profundas e, muitas 
vezes sem volta, para questões fundamentais como a distribuição do poder político e econômico, a crise ambiental, o câmbio climático, a distribuição internacional da produção e do consumo, do emprego e da renda, tudo isto com reconhecida e definitiva influência sobre a situação da saúde das populações humanas.

Por esta razão, parece importante que seja estabelecida ampla mobilização, no plano dos países e na esfera internacional, da sociedade civil - aí incluidas as Universidades e Institutos de pesquisa, representações de trabalhadores e outras institutições - para que seus interesses, visões e propostas apareçam na agenda do desenvolvimento sustentável para depois de 2015.

A última orientação quanto ao ODS Saúde que se origina do OWG - reforça a visão ampliada de saúde, quando define "assegurar vidas saudáveis e bem-estar" para todos em todas as idades". Entretanto, as metas propostas e os meios de implementação descolam do objetivo maior, estreitando seu alcance, por abordar metas exclusivamente voltadas para a atenção à saúde individual, sem valorizar a promoção da saúde, com poucas metas (e muito estreitas) em prevenção e, ademais, omitindo elementos importantes para o objetivo a que se propõe, que se encontram no campo da saúde pública / saúde coletiva, entre as quais as vigilâncias epidemiológica, sanitária

\section{Resumen}

El artículo examina la presencia de la salud como $\mathrm{Ob}$ jetivo de Desarrollo Sostenible (ODS) en la Agenda de Desarrollo post 2015, en el proceso transcurrido entre 2012 y 2014. Presente desde la Cumbre del Milenio y los ODM (2000), la salud también aparece en los documentos que tratan sobre la Agenda post 2015, desde Rio+20 hasta el documento del Grupo de Trabajo Abierto de Naciones Unidas (OWG), sometido a la Asamblea General de Naciones Unidas (AGNU) 2014-2015, pasando por la Consulta Global sobre Salud y el Grupo de Alto Nivel de Personas Eminentes. Los Autores concuerdan en la uniformidad con que la salud aparece en estos documentos. Identifican la amplitud del enunciado del ODS Salud, pero advierten sobre el fraccionamiento y la reducción conceptual de sus metas. Proponen cambios en las mismas, acrecentando la cuestión de los determinantes sociales de la salud y metas en salud pública, ausentes en la propuesta del OWG. Alertan sobre la necesidad tanto de reformas en la gobernanza global y nacional, como de la participación de la sociedad civil y de la influencia que puede ejercer sobre la diplomacia, responsable del pacto que será firmado en la AGNU de 2015.

Prioridades en Salud; Desarrollo Sostenible; Salud Mundial e ambiental, e o enfrentamento das inequidades e dos determinantes sociais da saúde. Por esta razão, propõe-se as alterações nas metas do ODS Saúde propostas na Figura 4.

As propostas de governança global e nacional são muito débeis e incompletas para as dificuldades inerentes a um processo desta envergadura e complexidade. Será preciso empreender profunda reformulação das metas e dos meios de implementação para garantir coerência e capacidade de concretização do ambicioso enunciado do ODS Saúde, evitando que se repitam as mesmas limitações encontradas na Agenda do Milênio com seus ODM Saúde.

Em um nível mais geral, as governanças nacionais e global precisam ser transformadas com vistas à implementação de planos de desenvolvimento coerentes com a equidade e a inclusão social, num contexto de desenvolvimento econômico que preserve o meio ambiente e os recursos naturais ameaçados.

Finalmente, entende-se que na redefinição do papel do Estado como provedor de direitos fundamentais como a saúde e outros ODS e no planejamento e ação intersetorial sob a égide do poder público, nos planos nacionais e globais, se encontre a chave para o desenvolvimento sustentável e a consecução dos ODS.

\section{Colaboradores}

P. M. Buss redigiu as versões preliminares e final. Os demais autores contribuíram com comentários ao texto. 


\section{Referências}

1. United Nations. Millennium declaration. http:// www.un.org/millennium/declaration/ares552e. pdf (acessado em 20/Jul/2014).

2. United Nations. The future we want. http://www. un.org/ga/search/view_doc.asp?symbol=A/RES/ 66/288\&Lang (acessado em 20/Jul/2014).

3. United Nations. Health in the post-2015. Agenda: report of the global thematic consultation on health. http:/ /www.worldwewant2015.org/file/337378/ download/366802 (acessado em 20/Jul/2014).

4. United Nations. The report of the high-level panel of eminent persons on the post-2015 development agenda: a new global partnership - eradicate poverty and transform economies through sustainable development. http://www.post2015hlp.org/wpcontent/uploads/2013/05/UN-Report.pdf (acessado em 20/Jul/2014).

5. United Nations. The Sustainable Development Solutions Network. Health in the framework of sustainable development. Technical report for the post-2015. http://unsdsn.org/resources/publica tions/health-in-the-framework-of-sustainabledevelopment/ (acessado em 20/Jul/2014).

6. United Nations. Open working group on sustainable development goals. http://sustainabledevel opment.un.org/focussdgs.html (acessado em 20/ Jul/2014).

7. Kickbusch I, Brindley C. Health in the post-2015 development agenda. An analysis of the UN-led thematic consultations, high-level panel report and sustainable development debate in the context of health. http://apps.who.int/iris/bitstream/10 665/85535/1/9789241505963_eng.pdf (acessado em 20/Jul/2014).

8. Laurell AC. Sistemas universales de salud: retos y desafios. http://www.isags-unasur.org/biblioteca interna.asp?lang=1\&idArea=33\&idPai=5712 (acessado em 20/Jul/2014).

9. Legge D. Health in the post-2015 development agenda. Third World Resurgence 2014; 284:45-8.

10. United Nations. The Millennium Development Goals report. http://www.un.org/millennium goals/2014\%20MDG\%20report/MDG\%202014\%20 English\%20web.pdf (acessado em 25/Out/2014).

11. The Economic and Social Council, United Nations. Addressing ongoing and emerging challenges for meeting the Millennium Development Goals in 2015 and for sustaining development gains in the future, 2014. http://daccess-dds-ny.un.org/ $\mathrm{doc} / \mathrm{UNDOC} / \mathrm{GEN} / \mathrm{N} 14 / 303 / 70 / \mathrm{PDF} / \mathrm{N} 1430370$ pdf?OpenElement (acessado em 25/Out/2014).

12. United Nations; World Trade Organization. Agreement on trade-related aspects of intellectual property rights. http://www.wto.org/english/tratop_e/ trips_e/t_agm0_e.htm (acessado em 20/Jul/2014).

13. United Nations. High-Level Political Forum. First meeting. http://www.un.org/ga/search/view_doc. asp?symbol=A/69/25\&Lang=E (acessado em 25/ Out/2014).

14. United Nations. High-Level Political Forum. 2nd meeting. http://www.un.org/ga/search/view_doc. asp?symbol=E/2014/L.22\&Lang=E (acessado em 25/Out/2014).
15. United Nations. Intergovernmental Committee of Experts on Sustainable Development Financing. Final draft (Advance Unedited Version, 8 August 2014). http://sustainabledevelopment.un.org/ content/documents/4588FINAL\%20REPORT\%20 ICESDF.pdf (acessado em 04/Set/2014).

16. Chasek P. The Regional Implementation Meetings for Rio+20 follow-up: an analysis of the outcomes. http://uncsd.iisd.org/policy-updates/the-regional-implementation-meetings-for-rio20-follow-upan-analysis-of-the-outcomes/ (acessado em 25/ Out/2014).

17. Conferencia de las Naciones Unidas sobre el Desarrollo Sostenible en América Latina y el Caribe. Documento preliminar. http://www.eclac.cl/ rio20/noticias/paginas/6/48936/2013-123-sus tainable_development_WEB.pdf (acessado em 25/ Out/2014).

18. United Nations. The world we want. Thematic consultations. http://www.worldwewant2015.org/ sitemap\#thematic (acessado em 20/Jul/2014).

19. United Nations. The Sustainable Development Solutions Network. Health In the framework of sustainable development. Technical report for the post-2015. http://unsdsn.org/resources/publica tions/health-in-the-framework-of-sustainabledevelopment/ (acessado em 20/Jul/2014).

20. Ministério das Relações Exteriores. Grupo de Trabalho Interministerial sobre a Agenda para o Desenvolvimento Pós-2015. Diplomacia pública, nota 76. http://www.itamaraty.gov.br/sala-de-imprensa/notas-a-imprensa/lancamento-do-grupo-detrabalho-interministerial-sobre-a-agenda-parao-desenvolvimento-pos-2015-palacio-itamaraty31-de-marco-de-2014 (acessado em 20/Jul/2014).

21. Group of 77 and China. Common positions on means of implementation for SDGs. http://sustai nabledevelopment.un.org/content/documents/ 4209G77\%20common\%20position\%20MOI.pdf (acessado em 20/Jul/2014).

22. United Nations. Open Working Group. Final compilation of amendments to goals and targets by major groups and other stakeholders including citizen's responses to MY World 6 priorities. http:// sustainabledevelopment.un.org/content/docume nts/4438mgscompilationowg13.pdf (acessado em 25/Out/2014).

23. Victora CG, Buffler P, Ebrahim S, Mandil A, Olsen J, Pearce N, et al. A position paper by the International Epidemiological Association. http://www. worldwewant2015.org/node/292903 (acessado em 20/Jul/2014).

24. International Conference on Population and Development. Policy recommendations for the ICPD beyond 2014: sexual and reproductive health \& rights for all. http://www.icpdtaskforce.org/beyond-2014/policy-recommendations.html (acessado em 20/Jul/2014).

25. World Health Organization. Universal health coverage. http://www.who.int/features/qa/universal_ health_coverage/en/ (acessado em 25/Out/2014).

26. World Bank. World development report 1993 investing in health. New York: Oxford University Press; 1993. 
27. Buss PM, Machado JMH, Gallo E, Magalhães DP, Setti AFF, Franco Netto FA, et al. Governança da saúde e ambiente no desenvolvimento sustentável. Ciênc Saúde Coletiva 2012; 17:1479-91.

28. Organização Mundial da Saúde. Comissão para os Determinantes Sociais da Saúde. Redução das desigualdades no período de uma geração. Igualdade na saúde através da acção sobre os seus determinantes sociais. Relatório final da Comissão para os Determinantes Sociais da Saúde. http:// cmdss2011.org/site/wp-content/uploads/2011/ 07/Redução-das-Desigualdades-no-período.pdf (acessado em 20/Jul/2014).

29. World Health Organization. World Conference on Social Health Determinants. Declaração política do Rio sobre determinantes sociais da saúde. http:// cmdss2011.org/site/wp-content/uploads/2011/12/ Decl-Rio-versao-final_12-12-2012.pdf (acessado em 20/Jul/2014).
30. World Health Organization. Health in all policies: Helsinki statement. Framework for country action. http://www.who.int/healthpromotion/ conferences/8gchp (acessado em 20/Jul/2014).

31. Ottersen OP, Dasgupta J, Blouin C, Buss P, Chongsuvivatwong V, Frenk J, et al. The Lancet-University of Oslo Commission on Global Governance for Health. The political origins of health inequity: prospects for change. Lancet 2014; 383:630-67.

32. Division for Sustainable Development, United Nations. HLPF issue briefs 5: from silos to integrated policy making. http://sustainabledevelopment. un.org $/$ index.php?page $=$ view $\&$ type $=400 \& n r=1322$ \&menu=35 (acessado em 20/Jul/2014).

Recebido em 17/Set/2014

Versão final reapresentada em 30/Out/2014

Aprovado em 30/Out/2014 